\title{
DISCUSSION
}

\section{Theoretical analysis of centrifugal model tests on reinforced earth structures}

\author{
A. SAWICKI (1998). Géotechnique 48, No. 4, 563-567
}

\section{E. Ausilio and E. Conte, University of Calabria}

The author proposes the use of some equations to predict the acceleration scale factor of centrifugal model tests on reinforced earth structures. These equations have been derived under the assumption that the failure surface is planar and makes an angle of $\alpha=45^{\circ}-\phi / 2$ with respect to the vertical. Several tests conducted on reduced-scale models and centrifugal models of reinforced earth structures have, however, demonstrated that the assumption of a log-spiral or circular failure surface is more realistic than that of a plane surface. In particular, the results published recently by Zornberg et al. (1998) show that failure is characterised by a well-defined curved surface; it initiates near the mid-height of the structure and occurs without evidence of pull-out.

In order to obtain a more suitable solution for the centrifugal acceleration scale factor, and also to provide an effective means of comparing the results presented by the author, the upperbound theorem of limit analysis is here applied referring to a log-spiral surface discontinuity mechanism (Fig. 6). The case of global collapse of the structure is considered when a uniform distribution with depth of the maximum reinforcement forces may be assumed (Zornberg et al., 1998). Following the kinematic approach of limit analysis, the upper-bound solution for the centrifugal acceleration scale factor at failure is found:

$$
N_{\mathrm{f}}=\frac{1}{2} \frac{\sigma_{\mathrm{o}}}{\gamma_{\mathrm{o}} H} F\left(\theta_{\mathrm{o}}, \theta_{\mathrm{h}}, \beta, \phi\right)
$$

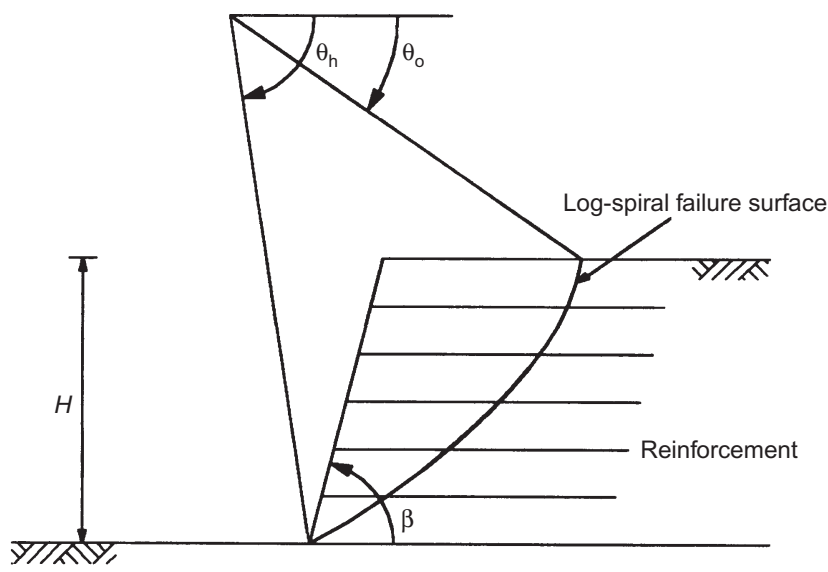

Fig. 6. Configuration of a reinforced earth structure with log-spiral failure surface where

$$
\begin{aligned}
& F\left(\theta_{\mathrm{o}}, \theta_{\mathrm{h}}, \beta, \phi\right) \\
& \quad=\frac{\left[\sin ^{2} \theta_{\mathrm{h}} \mathrm{e}^{2\left(\theta_{\mathrm{h}}-\theta_{\mathrm{o}}\right) \operatorname{tg} \phi}-\sin ^{2} \theta_{\mathrm{o}}\right]\left[\sin \theta_{\mathrm{h}} \mathrm{e}^{\left(\theta_{\mathrm{h}}-\theta_{\mathrm{o}}\right) \operatorname{tg} \phi}-\sin \theta_{\mathrm{o}}\right]}{\left(f_{1}-f_{2}-f_{3}\right)}
\end{aligned}
$$

and $f_{1}, f_{2}$ and $f_{3}$ are functions of $\phi$, and of the angles $\theta_{\mathrm{o}}, \theta_{\mathrm{h}}$ and $\beta$ indicated in Fig. 6 (Chen, 1975). In deriving equation (19), the energy dissipation rate in the reinforcement has been determined by the expression proposed by Michalowski (1997).

We have used equation (9) in conjunction with an optimisation procedure to obtain the least upper-bound for $N_{\mathrm{f}}$ of two reinforced retaining wall models. The results are shown in Table 1 , where the last two lines concern an ideal wall model, which is assumed to have lower values of $\phi$ than those of the other cases. The values of $N_{\mathrm{f}}$ calculated by equation (9) are also presented in the table for comparison. As can be noted, equation (9) gives values of $N_{\mathrm{f}}$ that are higher than those obtained applying the upper-bound theorem of limit analysis. The difference between the results increases as the value of $\phi$ decreases. Nevertheless, in the cases examined the differences in excess are $10 \%$ at the most. In connection with practical problems, the differences found are not too much: therefore equation (9), besides being very easy to use, may come in handy to predict the acceleration scale factor $N_{\mathrm{f}}$ corresponding to the global failure of reinforced retaining wall models in centrifugal tests, especially when the value of $\phi$ is high.

\section{Author's reply}

Equation (9), similarly to equation (19), also corresponds to the upper-bound estimate of the centrifugal acceleration scale factor. The basic difference is that equation (9) has been derived for the planar failure surface, which has been assumed as the most simple geometry of the structural collapse. I agree that in the case of reinforced slopes (RSs) a more complex shape of the failure surface (log-spiral, bilinear, etc.) should be assumed, but for the RS retaining walls a planar character of this surface is justified by available empirical data.

An obvious advantage from assuming a planar failure surface is simplicity of the theory describing the centrifugal model tests, and subsequently simple, analytical formulae, which may be used in engineering calculations. This is similar to some other assumptions in structural mechanics and strength of materials. For example, the assumption about the planar character of a beam's cross-sections has resulted in a very simple theory of beams, widely applied in civil engineering. I am glad that the discussers have noticed a simplicity of equation (9) and propose this equation as an useful engineering tool. Their verification of this equation against the other, but much more

Table 1. $N_{\mathrm{f}}$ values for two reinforced retaining wall models

\begin{tabular}{c|c|c|c|c|c|c}
\hline$H: \mathrm{mm}$ & $\gamma_{\mathrm{o}}: \mathrm{kN} / \mathrm{m}^{3}$ & $\sigma_{\mathrm{o}}: \mathrm{kN} / \mathrm{m}^{2}$ & $\phi:$ degrees & $N_{\mathrm{f}}:$ eq $(19)$ & $N_{\mathrm{f}}:$ eq $(9)$ & Reference \\
\hline 202 & $16 \cdot 9$ & $62 \cdot 54$ & $45 \cdot 8$ & 210 & $92 \cdot 5$ & 222 \\
202 & $16 \cdot 9$ & $29 \cdot 43$ & $44 \cdot 5$ & 96 & 98 \\
202 & $16 \cdot 9$ & $31 \cdot 27$ & 44 & 102 & Bolton \& Pang (1982) \\
508 & $18 \cdot 4$ & $15 \cdot 70$ & 42 & 50 & 17 \\
202 & $16 \cdot 9$ & $25 \cdot 00$ & 35 & 54 & $*$ \\
202 & $16 \cdot 9$ & $25 \cdot 00$ & 30 & 40 & Jaber \& Mitchell (1990) & $*$ \\
\hline
\end{tabular}

*Ideal wall model 
complicated, upper-bound estimate is also valuable. Equation (19), which gives lower values of the centrifugal acceleration scale factor at failure, is obviously more 'exact' than equation (9) from the viewpoint of limit-state theory, because it gives results that are closer to the unknown, exact solution of the theoretical problem. It does not mean that the exact theoretical solution of the limit-state problem (if known!) should always coincide with experimental data. It seems that the differences in $N_{\mathrm{f}}$, shown in Table 1 are quite acceptable.

The theory presented in my technical note makes possible an analysis of the whole process of structural failure, from its initiation, through propagation, up to the development of the mechanism of failure. Also, equation (19) defines only the final stage of the RS structure behaviour, and the approach proposed by the discussers does not allow for the study of the stages preceding the global failure. Equation (19) is more general, since it also facilitates the analysis of reinforced slopes, while equation (9) deals with RS retaining walls exclusively. However, the theory proposed can be extended to the case of reinforced slopes, for more complex shapes of the failure surface.

Determination of the point at which the failure is initiated is an essential element of such an extended theory. In the case of RS retaining walls, investigated in a geotechnical centrifuge, the failure is usually initiated at the toe of the structure. Probably the same is true for steep reinforced slopes. The arguments of Zornberg et al. (1998), that the failure is initiated in the middle of the slope, are not convincing because they did not measure the strains in reinforcement, but performed only a simplistic visual analysis of the failure's initiation (see their Fig. 9a).
Moreover, they did not pay any attention to the fact that there was a double amount of reinforcement at the failure surface near the slope's toe, owing to the reinforcement's overlap (see their Fig. 14). Therefore the slope was stronger in its lower part than in the upper part. In such a case, the failure may indeed be initiated at a point lying outside this 'double-reinforced' zone; but this is another problem of non-uniformly reinforced slopes. The model reinforced slopes investigated by Zornberg et al. (1998) were rather distant from theoretically oriented, readable statical schemes. Nor can I agree with the 'alternative distribution of maximum reinforcement tensions' proposed by Zornberg et al. (1998) in their Fig. 15, which is obviously wrong.

Zornberg et al. (1998) applied a sufficiently long reinforcement to prevent its pull-out. In each particular case the problem of pull-out should be studied carefully because various experiments are performed in different conditions.

\section{REFERENCES}

Chen, W. F. (1975). Limit analysis and soil plasticity. Amsterdam: Elsevier.

Jaber (1990) cited in text.

Michalowski, R. L. (1997). Stability of uniformly reinforced slopes. J. Geotech. and Geoenvir. Engng, ASCE 123, No. 6, 546-556.

Zornberg, J. G., Sitar, N. \& Mitchell, J. K. (1998). Performance of geosynthetic reinforced slopes at failure. J. Geotech. and Geoenvir. Engng, ASCE 124, No. 8, 670-683. 